\title{
流动化学在卤化反应中的应用
}

\author{
刘玎 $a, b$ 朱园园 $c$ 古双喜*, $a, b$ 陈芬儿*,a,b,d \\ ( ${ }^{a}$ 武汉工程大学化工与制药学院 绿色化工过程教育部重点实验室 武汉 430205) \\ ( ${ }^{b}$ 武汉工程大学药物研究院 武汉 430205) \\ ( ${ }^{c}$ 武汉工程大学化学与环境工程学院 武汉 430205) \\ ( ${ }^{d}$ 复旦大学化学系 上海 200433)
}

\begin{abstract}
摘要 有机物的卤化反应是有机合成中最重要的转化之一. 传统釜式卤化反应存在高放热及选择性差等问题，且卤化 试剂一般具有毒性和腐蚀性. 流动化学在传质和传热方面具有显著优势, 可精确控制反应温度及试剂用量, 并且可在 线淬灭危险试剂, 避免其暴露. 按有机化合物卤化反应分类, 系统地归纳了流动化学在氟化反应、氯化反应、溴化反应 和碘化反应中的应用进展，并展望了其发展趋势.
\end{abstract}

关键词 流动化学; 微反应器; 连续流; 卤化; 有机合成

\section{Application of Flow Chemistry in Halogenation}

\author{
Liu, Ding ${ }^{a, b}$ Zhu, Yuanyuan ${ }^{c} \quad$ Gu, Shuangxi ${ }^{*, a, b} \quad$ Chen, Fener ${ }^{*, a, b, d}$ \\ ( ${ }^{a}$ Key Laboratory for Green Chemical Process of Ministry of Education, School of Chemical Engineering and Pharmacy, \\ Wuhan Institute of Technology, Wuhan 430205) \\ ( ${ }^{b}$ Pharmaceutical Research Institute, Wuhan Institute of Technology, Wuhan 430205) \\ ( ${ }^{c}$ School of Chemistry and Environmental Engineering, Wuhan Institute of Technology, Wuhan 430205) \\ ( ${ }^{d}$ Department of Chemistry, Fudan University, Shanghai 200433)
}

\begin{abstract}
The halogenation of organic compounds is one of the most important transformations in organic synthesis. However, there are some problems in traditional batch halogenation, such as high exothermicity, poor selectivity and the use of highly toxic and corrosive halogenating agents. Flow chemistry has its unique advantages such as high mixing efficiency, fast heat and mass transfer performance, accurate control over process parameters and enhanced process safety, reduced energy input and so on. According to the classification of halogenation of organic compounds, the main progress of flow chemistry in fluorination, chlorination, bromination and iodization is summarized systematically, and its development trend is prospected. Keywords flow chemistry; microreactor; continuous flow; halogenation; organic synthesis
\end{abstract}

有机卤化物是含有氟、氯、澳或碘等卤族元素的有 机化合物，因其特有的物理性能、化学反应活性和生理 学功能, 在医药、农药、化工及材料等众多领域作为产 品、合成中间体及溶剂等发挥着广泛而重要的作用 ${ }^{[1]}$. 卤化反应是制备有机卤化物的主要方法. 即使是使用含 卤素的中间体来合成目标卤代分子, 追根溯源, 这些中 间体也要通过直接卤化来获得 ${ }^{[2]}$. 卤化反应中常使用的 卤化试剂如卤素 $\left(\mathrm{X}_{2}\right)$ 和氢卤酸 $(\mathrm{HX})$ 等一般具有高反应 性、刺激性和腐蚀性, 且反应一般为剧烈的放热反应,
反应迅速，难以控制，选择性较差 ${ }^{[3]}$. 而目前的卤化工 艺一般是通过传统釜式反应来进行，存在反应不易控 制，传质传热慢，浓度和热量分布不均，人工操作时间 长, 易与有害试剂接触等缺点, 易引发安全问题 ${ }^{[4]}$. 因 此, 利用新技术更加高效、安全地进行卤化反应具有重 大意义.

流动化学, 亦称为连续流或柱塞流化学, 其主要特 征是在连续流动的流体中进行化学反应. 连续流微反应 技术作为利用集成的单元微反应器(微混合器、微换热

\footnotetext{
* Corresponding authors. E-mail: shuangxigu@163.com; rfchen@fudan.edu.cn Received July 22, 2020; revised September 14, 2020; published online October 10, 2020.

Project supported by the National Natural Science Foundation of China (Nos. 21877087, 21602164) and the Open Fund of Hubei Key Laboratory of Novel Reactor and Green Chemical Technology (Wuhan Institute of Technology) (No. K202004).

国家自然科学基金(Nos. 21877087, 21602164)和新型反应器与绿色化学工艺湖北省重点实验室(武汉工程大学)开放基金(No. K202004)资助项目.
} 
器、微分离器和微控制器等) 来进行流动化学反应的一 种技术, 通过百输送物料, 然后以连续流动模式进行反 应 $^{[5]}$. 常用的连续流微反应器包括微通道反应器、毛细 管微反应器、降膜式微反应器、多股并流式微反应器和 膜分散式微反应器等. 微通道反应器是其中使用最为广 泛的微反应器, 通过精细加工技术(光刻、蚀刻和机械加 工等)可以在硅、玻璃或聚合物(聚甲基丙烯酸甲酯等) 等材料上制作尺寸各异的微通道. 玻璃、硅和陶瓷制成 的微反应器生成成本高, 兼容性较差, 大多用于简单的 化学合成工艺, 可突出小规模连续流操作的特点; 而以 不锈钢管或全氟聚合物为材料的微反应器生产成本较 低, 可使连续流微反应器的应用快速大规模扩展. 可针 对化学反应的特点, 如温度、压力、腐蚀性及比热容等, 选择合适的微反应器制作材料 ${ }^{[6]}$. 近年来, 流动化学发 展迅速, 在药物合成 ${ }^{[7]}$ 、不对称合成 ${ }^{[8]}$ 、多相反应 ${ }^{[9]}$ 、光 化学反应 ${ }^{[10]}$ 和电化学反应 ${ }^{[11]}$ 、涉及高活性和危险试剂的 反应 ${ }^{[12]}$ 等有机合成反应中应用日趋广泛. 流动化学因 其传质传热效率高、过程重复性好、连续自动化操作和 时空效率高、产品质量稳定和安全系数高等优点, 可克 服传统卤化反应中存在的问题. 本文主要介绍流动化学 在卤化反应中的应用、目前存在的挑战和未来的发展前 景.

\section{1 流动化学与卤化反应}

流动化学反应所使用的微反应器尺寸小(内部通道 尺寸通常为 $10 \sim 1000 \mu \mathrm{m}$ ), 反应物在管道中扩散距离 短, 可精确控制进料的质量、传热和停留时间 ${ }^{[13]}$. 与传 统釜式反应技术相比, 流动化学主要有以下特点 ${ }^{[14]}$ : (1)反应速率快, 物料混合快, 传质传热效率高; (2)反应 过程易控制, 选择性高, 可实时监测反应条件(温度、压 力和进料速度等)和在线分析, 以保证实验过程的一致 性; (3)无工艺放大效应, 可通过并联反应器来实现更大 规模的生产; (4)反应持液量小, 进行高温高压反应安全 性更高; (5)物料在微反应器通道中传递速率高, 接触时 间短, 副产物少. 因此, 使用连续流微反应技术, 可以 安全、可控地进行卤素和卤化氢参与的卤化反应. 即使 是气液两相反应, 也能精确控制试剂的用量和反应放 热. 对于危险试剂, 可以进行在线淬灭从而避免不必要 的暴露，显著提高反应过程中的安全性.

\section{1 流动化学与氟化反应}

由于氟原子具有较小的原子半径和最强的电负性, 其引入通常会使有机化合物产生独特的物理和化学性 质，因而有机氟化物广泛存在于在医药、农药与材料 中 $^{[15]}$. 在药物研究与开发中, 氟原子不仅可以提高药物 的生物活性, 还可以改善药物的生物利用度、亲脂性和
代谢稳定性 ${ }^{[16]}$. 在 2018 年排名前 200 畅销药物中, 含有 氟原子的新化学实体数目就达到 34 个, 氟原子的重要 性可见一斑.

目前获得有机氟化物的方法包括：(1)直接氟化法, 使用亲电、亲核或自由基氟化剂在底物上直接引入氟原 子，常用的氟化试剂有 $F_{2} 、 H F$ 、二乙胺三氟化硫 (DAST)、 $N$-氟代双苯磺酰胺(NFSI)和 1-氯甲基-4-氟-1,4重氮化二环 2.2.2 辛烷双 (四氟嗍酸)(Selectfluor) 等 ${ }^{[17]}$; (2) 将含氟中间体作为合成砌块来合成目标产物, 不涉 及 $\mathrm{C}-\mathrm{F}$ 键的断裂与形成, 比直接氟化的反应条件更温 和. 引入含氟砌块的方法主要是对底物进行氟烷基化, 包括三氟甲基化和全氟烷基化等 ${ }^{[18]}$.

直接氟化法的原子效率高, 成本低, 但使用的试剂 活性高且反应高度放热, 难以控制. 研究发现, 将流动 化学应用于直接氟化过程能显著提高反应的选择性和 安全性. Chambers 研究组 ${ }^{[19]}$ 于 1999 年首次报道了利用 连续流微反应技术直接对 $\beta$-二羰基化合物进行氟化的 工作。该实验选用氟气作为氟化剂, 通过一种用镍聚氯 三氟乙烯封装的(或铜)微薄膜反应器，将反应物溶液用 注射百注入, 氟气通过流量控制器与基板液体溶液反 应，该反应的氟化转化率高达 $99 \%$, 收率为 $73 \%$ (Scheme 1).

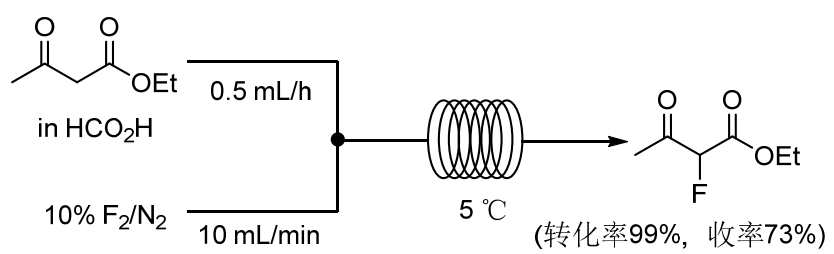

图式 $1 \beta$ 二二羰基化合物的氟化

Scheme 1 Fluorination of $\beta$-dicarbonyl compounds

Chambers 研究组 ${ }^{[20]}$ 于 2000 年在三通道微薄膜反应 器中使用氟气对 4-甲氧基硝基苯直接氟化, 使用乙腈或 乙腈/甲酸混合物作为溶剂, 反应温度为 $0 \sim 5{ }^{\circ} \mathrm{C}$, 可减 少氟气与溶剂之间的反应. 所有的产物经过层析柱的纯 化和分离. 产生的二氟化副产物比传统间歇过程的少, 因为单氟化产物可直接流出通道, 防止进一步氟化. 具 体路线见 Scheme 2.

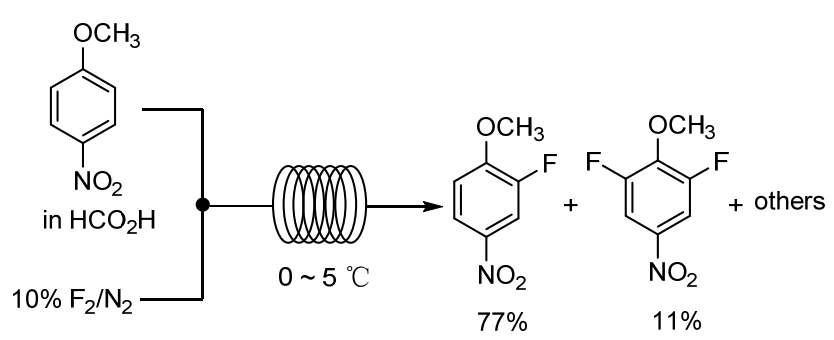

图式 2 4-甲氧基硝基苯的氟化

Scheme 2 Fluorination of 4-methoxynitrobenzene 
Chambers 研究组 ${ }^{[21]}$ 于 2008 年报道了利用连续流动 技术直接氟化一系列苯甲醛衍生物, 结果表明, 产物的 生成与芳环上的取代基有关. 含有甲氧基等给电子取代 基的苯甲醛底物, 会进行亲电攻击而产生了环氟化结 构. 相反, 带有吸电子取代基的苯甲醛底物生成相应的 酰基氟. 将第一步用氟气氟化的粗产品混合物与 3,5-二 硝基苄基醇反应，得到苯甲酸酯. 相比分批处理方法， 使用连续流动反应器更易放大工艺, 通过特定的冷却通 道还能实现有效的热控制. 液相在微反应通道中有非常 大的比表面积, 将气体和液体试剂同时通入多个不锈钢 通道中, 可大规模合成氟化衍生物(比如, 在一个 9 通道 反应器中 $24 \mathrm{~h}$ 可生成 $100 \mathrm{~g}$ 氟化酮酸酯), 且无明显的放 大效应(Scheme 3).
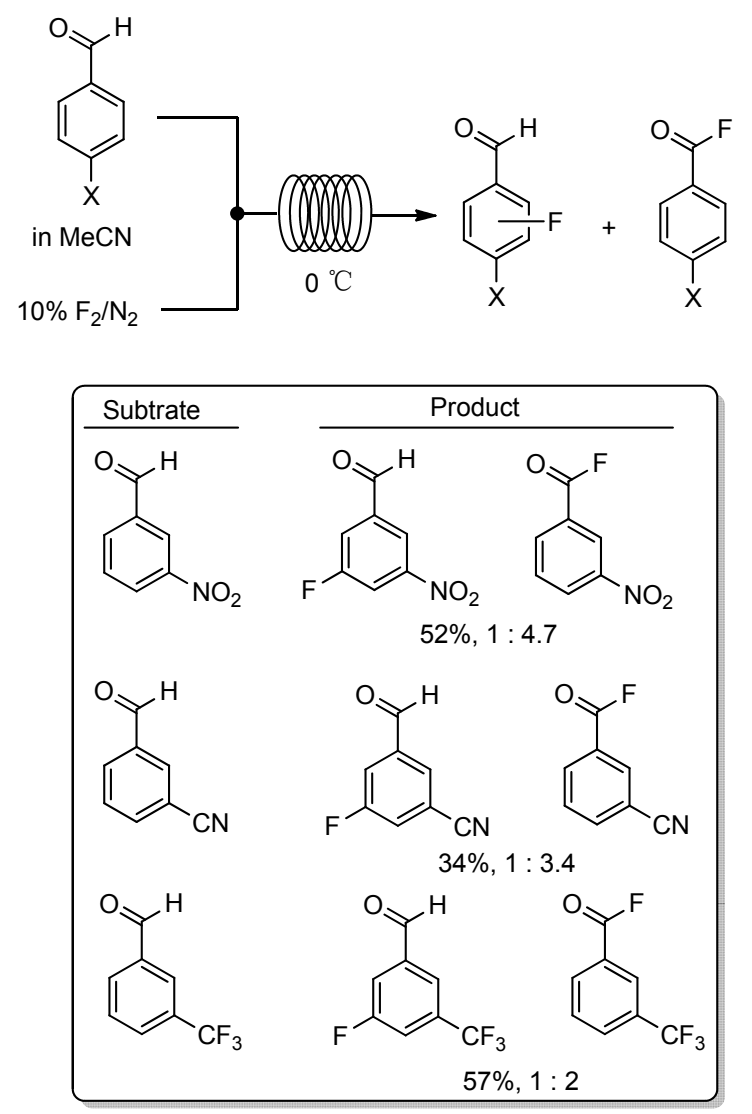

图式 3 苯甲醛衍生物的氟化

Scheme 3 Fluorination of benzaldehyde derivatives

Elgue 等 ${ }^{[22]}$ 报道了工业化规模上的 1,3-二羰基化合 物的氟化反应, 该反应在 Boostec 公司生产的 $\mathrm{SiC}$ 连续 流微反应器中进行. $\mathrm{SiC}$ 材料具有优异的传热能力和耐 腐蚀性, 非常适合大规模的高放热反应. 反应底物溶于 乙腈/醋酸 $(V: V=1: 1)$ 后与 $\mathrm{F}_{2} / \mathrm{N}_{2}$ 在气液两相连续流反 应器中反应, 实验结果表明, 底物可在短时间内完全转 化，目标产物的产率可达 $200 \mathrm{~g} \bullet \mathrm{h}^{-1}$.

传统釜式氟化反应中, 常用 DAST 作为氟化剂, 用
于醇、羰基等含氧有机物的氟化，但 DAST 反应条件严 苛，受热 $90{ }^{\circ} \mathrm{C}$ 以上会剧烈爆炸. 反应也常伴随副反应， 选择性较差. 使用连续流微反应器对反应进行温度和速 度控制, 可提高实验安全性和合成效率. Baumann 等 ${ }^{[23]}$ 于 2009 年采用 Vapourtec 公司生产的 R2+/R4 型连续流 微反应器，流管线圈由全氟烷氧基树脂等惰性材料制 成, 可扩大操作弹性和提高安全性, 设置自校准双永系 统. 将 DAST 和醇或羰基化合物在二氯甲烷溶剂中混 合, 经过 $\mathrm{T}$ 型混合器进入 $\mathrm{R} 4$ 单元的微管道中, 在 70 $90{ }^{\circ} \mathrm{C}$ 下进行 $27 \mathrm{~min}$ 左右的氟化反应, 再通过 $\mathrm{CaCO}_{3}$ 和 硅胶分离柱淬灭反应和捕捉氟化副产物, 得到的单氟化 产物收率一般大于 $80 \%$ (Scheme 4). 此外，文献还表明 该方法对各类不同底物(如苄醇、酰氯、环氧甲醇和烯 基醛等)均能适用, 多个产物的收率均达到 $95 \%$ 以上 ${ }^{[23]}$.
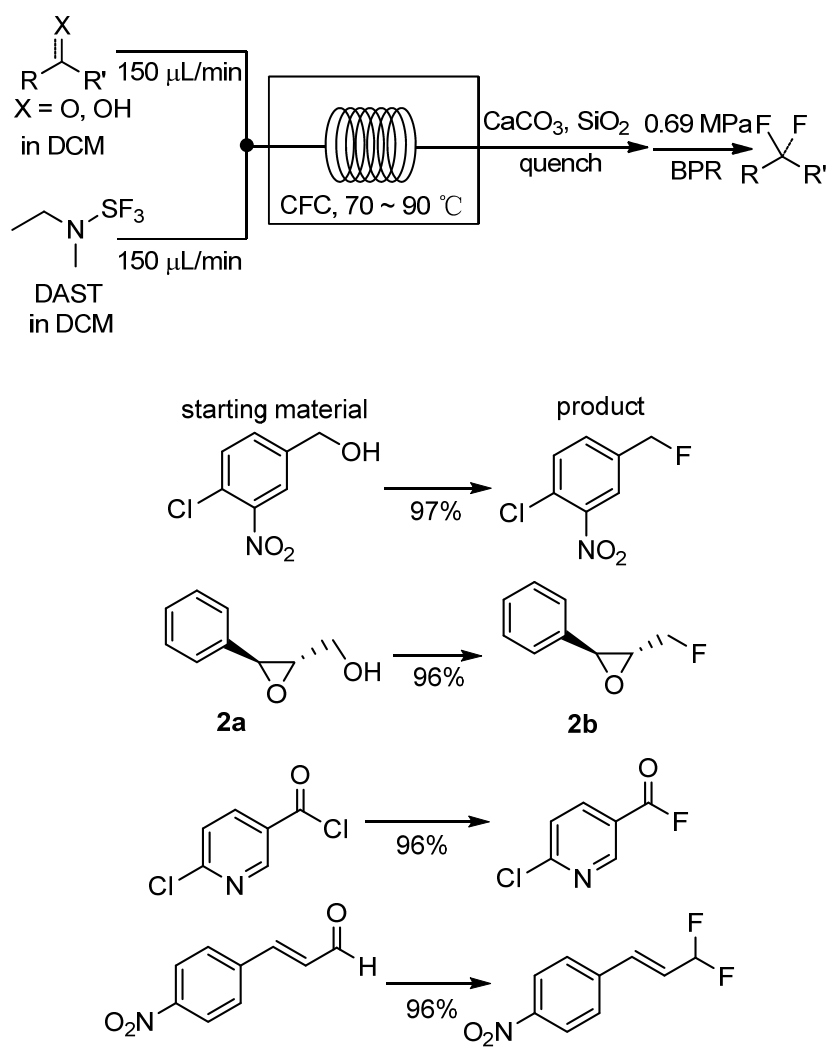

图式 4 在连续流微反应器中使用 DAST 氟化剂

Scheme 4 Use of DAST in continuous flow reactors

Nagaki 等 ${ }^{[24]}$ 报道了以取代溴苯或取代碘苯为原料 通过流动化学反应制备取代氟苯的方法. 在正丁基或苯 基锂的作用下，取代溴苯或取代碘苯发生卤一锂交换得 到芳基锂，进而在亲电氟化试剂(如 NFSI、 $N$-氟磺酰胺 等)作用下于微反应系统(由两个 $\mathrm{T}$ 型不锈钢微混合器和 两个不锈钢微管反应器 $\mathrm{R} 1 、 \mathrm{R} 2$ 组成)中反应得到氟化产 物。该反应方法对具有多种不同给电子和吸电子取代基 的底物均适用(Scheme 5) ${ }^{[24]}$. 正丁基锂和苯基锂等金属 
试剂在空气中易自燃, 采用传统的有机合成方法易引发 火灾; 而且该类化合物的高反应性会导致其在工业应用 中易发生爆炸. 连续流微反应技术可以很好地解决这些 问题，在实验室合成和工业化应用中都极具前景.

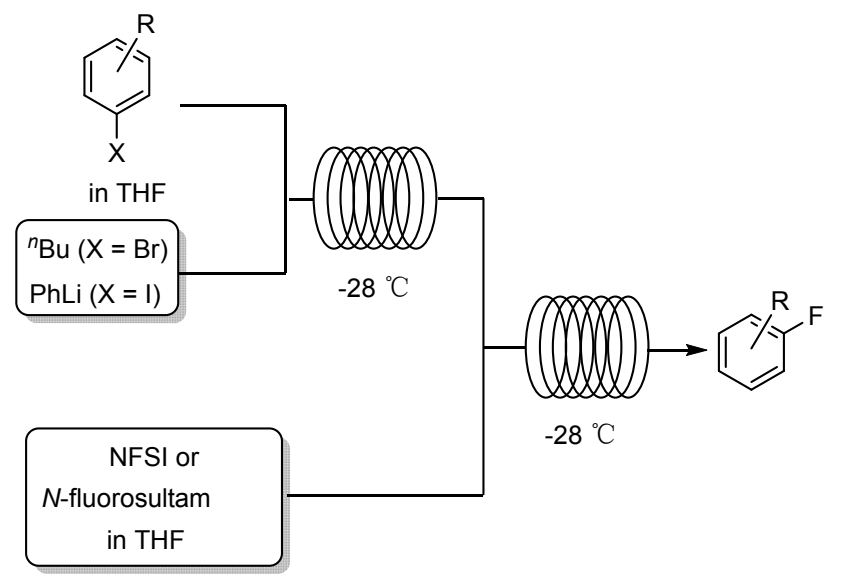

\begin{tabular}{l} 
Yield/\% \\
N-fluorosultam \\
\hline
\end{tabular}

图式 5 取代苯基锂的连续流氟化

Scheme 5 Continuous flow fluorination of substituted phenyl lithium

\section{2 流动化学与氯化反应}

有机氯化物在农药及医药领域用途非常广泛, 既用 作终端产品也用作中间体 ${ }^{[25]}$. 有机物的氯化反应一般 包括置换氯化、加成氯化和氧化氯化. 目前常用的氯化 剂有 $\mathrm{Cl}_{2} 、 \mathrm{HCl} 、 \mathrm{SOCl}_{2}$ 或 $\mathrm{PCl}_{5}$ 等.

$\mathrm{Cl}_{2}$ 是一种常用的氯化剂和氧化剂, 廉价易得. 但 $\mathrm{Cl}_{2}$ 反应活性高, 反应时会大量放热, 难以控制, 因此其 应用受限 ${ }^{[26]}$. 此外, 在有机合成实验室和生产设施中对 氯气瓶的处理也是一个重要的安全问题. $\mathrm{Cl}_{2}$ 的使用、处 理和运输有严格的规定, 不仅使用场所需要隔离, 还需
配备专业人员. 为解决这一问题, Kappe 等 ${ }^{[27]}$ 于 2016 年 开发了一种基于 $\mathrm{NaOCl}$ 和 $\mathrm{HCl}$ 反应的连续流氯气发生 器. 该反应器设有三个进料百输送三种物料 $(\mathrm{NaOCl}$ 水 溶液、水溶液和 $\left.\mathrm{CHCl}_{3}\right)$. 水溶液混合后生成的 $\mathrm{Cl}_{2}$ 被萃 取至有机相. 然后经疏水膜液-液分离器将含 $\mathrm{Cl}_{2}$ 的有机 相从水相中分离出来. 实验对进料流速进行了优化, 当 $\mathrm{NaOCl}$ 流速为 $100 \mu \mathrm{L} \cdot \mathrm{min}^{-1} 、 \mathrm{HCl}$ 流速为 $75 \mu \mathrm{L} \cdot \mathrm{min}^{-1}$ 、 $\mathrm{CHCl}_{3}$ 流速 $300 \mu \mathrm{L} \cdot \mathrm{min}^{-1}$ 时, $\mathrm{Cl}_{2}$ 产率最高, 为 $91 \%$. 实 验继续使用氯气发生器进行了几种有机反应的测试, 包 括氯一吡啶络合物对仲醇的选择性氧化以及硅烷和茮基 化合物的氯化反应, 均取得较好的结果(Scheme 6).

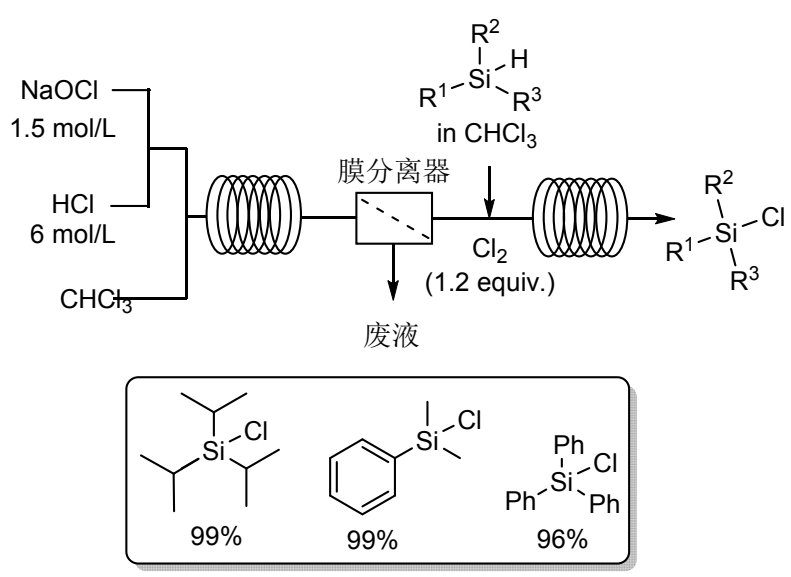

图式 6 硅烷的连续流氯化

Scheme 6 Continuous flow chlorination of silane

$\mathrm{NaOCl}$ 除了用于生成 $\mathrm{Cl}_{2}$, 还可直接用于胺的连续 氯化. Blacker 等 ${ }^{[28]}$ 于 2015 年报道了用次氯酸钠连续流 动合成 $N, N$-二烷基- $N$-氯胺. 将胺溶解在有机溶剂(如甲 苯)中，可使有机可溶产物与水溶性 $\mathrm{NaOH}$ 易于分离. 带 有静态混合器的管式反应器和连续摚拌槽式反应器都 能促进两相反应溶液和次氯酸钠水溶液的有效混合，原 位生成产物的效率可达 $72 \% \sim 100 \%$ ，停留时间为 3 20 $\mathrm{min}$, 产量约为 $0.05 \mathrm{~mol} / \mathrm{h}$. 该法比传统釜式反应能更好 地传质传热、减少副反应和提高收率.

$\mathrm{SOCl}_{2}$ (氯化亚砜) 常用于氯化烯烃、醇、羧酸等化合 物，包括合成常作为医药中间体的酰氯化合物. Movsisyan 等 ${ }^{[29]}$ 于 2018 年设计了一种以 $\mathrm{SOCl}_{2}$ 和丙烯酸 原料，连续流动合成 3 -氯丙酰氯，又进一步将 3 -氯丙酰 氯作为中间体合成贝克拉胺的反应. 实验采用双注射葲 将丙烯酸 [0.5 equiv. $N, N$-二甲基甲酰胺(DMF)] 和纯 $\mathrm{SOCl}_{2}$ 原入可熔性聚四氟乙烯(PFA)塑料管制成的反应 器并停留 $25 \mathrm{~min}$, 反应器在油浴中加热至 $80{ }^{\circ} \mathrm{C}$. 管式 反应器连接有背压调节器(BPR), 然后将反应混合物收 集在烧瓶中, 使用 $\mathrm{NaOH}$ 水溶液进行淬灭. 3 -氯丙酰氯 的产率为 $94 \%$, 通量可达 $4.5 \mathrm{~g} / \mathrm{h}$. 实验在上述反应基础 
上通过后续连续流反应步骤将 3 -氯丙酰氯转化为药物 活性成分贝克拉胺. 3-氯丙酰氯和茮胺分别被泵送通过 PFA 管式反应器, 停留时间为 $1 \mathrm{~min}$, 反应器在油浴中 加热至 $80{ }^{\circ} \mathrm{C}$. 产物收率为 75\% (Scheme 7) ${ }^{[29]}$. 该方法 原子经济性高且有直接放大生产的潜力.

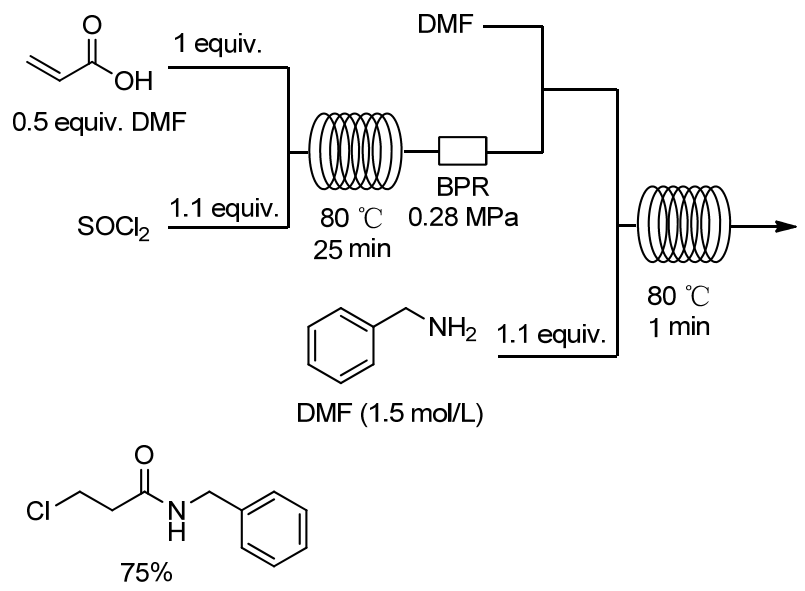

图式 7 以丙烯酸为原料合成 $3-$ 氯 $-N$ - 芐基丙酰胺

Scheme 7 Synthesis of 3-chloro- $N$-benzyl-propanamide from acrylic acid

醇的氯代是合成有机氯化物的重要方法之一，常见 的氯化剂有五氯化磷、三甲基乙酰氯、4-甲苯磺酰氯、 草酰氯和光气，但这些试剂通常有强刺激性和毒性，且 反应易产生副产物. 理想的绿色合成工艺是用氯化氢将 醇转化为氯化物, 原子经济性高, 唯一的副产物是水. Reichart 等 ${ }^{[30]}$ 于 2012 年报道了 $30 \%$ 盐酸在连续流微反应 器中对正丁醇、正己醇和正癸醇的氯化反应. 在高温高 压下，停留时间为 $15 \mathrm{~min}$, 对伯醇进行了高效的氯化脱 羟基反应. Borukhova 等 ${ }^{[31]}$ 于 2016 年直接使用无水氯化 氢气体, 将醇在 $100{ }^{\circ} \mathrm{C}$ 和 $1.0 \mathrm{MPa}$ 的压力下转化为相应 的氯化物(Scheme 8). 使用气体代替盐酸可使氯化氢从 3 equiv. 降至 1.2 equiv.. 例如, 在 $20 \mathrm{~min}$ 的停留时间内, 苯甲醇转化为氯化茮的产率为 $96 \%$. 改进的方法虽为气 液两相反应, 但在连续流微反应器中能实现良好的传质 传热和高反应收率; 与原法相比, 还可大大减少过量盐 酸的排放, 减少污染, 降低环保压力.

光氯化反应可应用于工业规模上氯化烃类化合物. 微反应器通道尺寸小, 在连续流反应时可对反应混合物 进行充分且均匀的照射. Fukuyama 等 ${ }^{[32]}$ 于 2016 年报道 了在 Kappe 课题组 ${ }^{[27]}$ 原位生成生成氯气技术的基础上, 对环烷烃、烷基苯和环己酮进行光氯化反应且获得了高 产率的氯化物. 实验采用 $352 \mathrm{~nm}$ 光源照射玻璃芯片反 应器, 反应后再用亚硫酸钠淬灭, 反应停留时间不超过 $1 \mathrm{~min}$. 经气相色谱分析，七种底物氯化的产率在 59\% $94 \%$, 具体路线见 Scheme $9^{[32]}$.

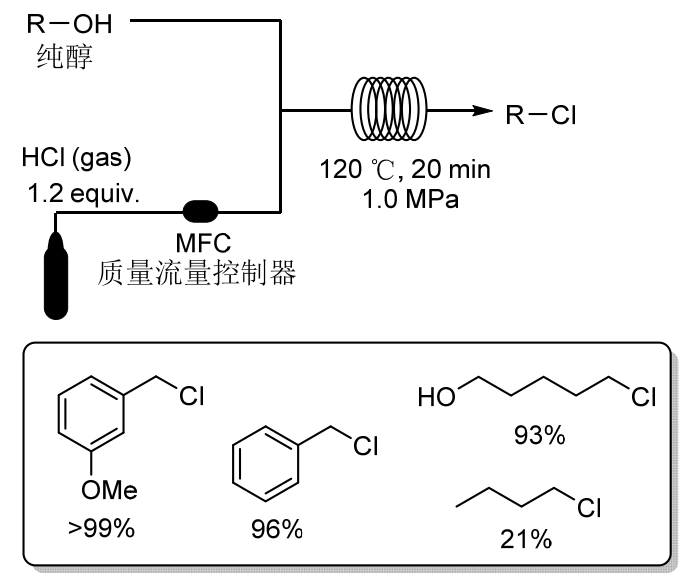

图式 8 使用 $\mathrm{HCl}$ 气体将醇转化为氯化物 Scheme 8 Conversion of alcohols to chlorides using $\mathrm{HCl}$ gas
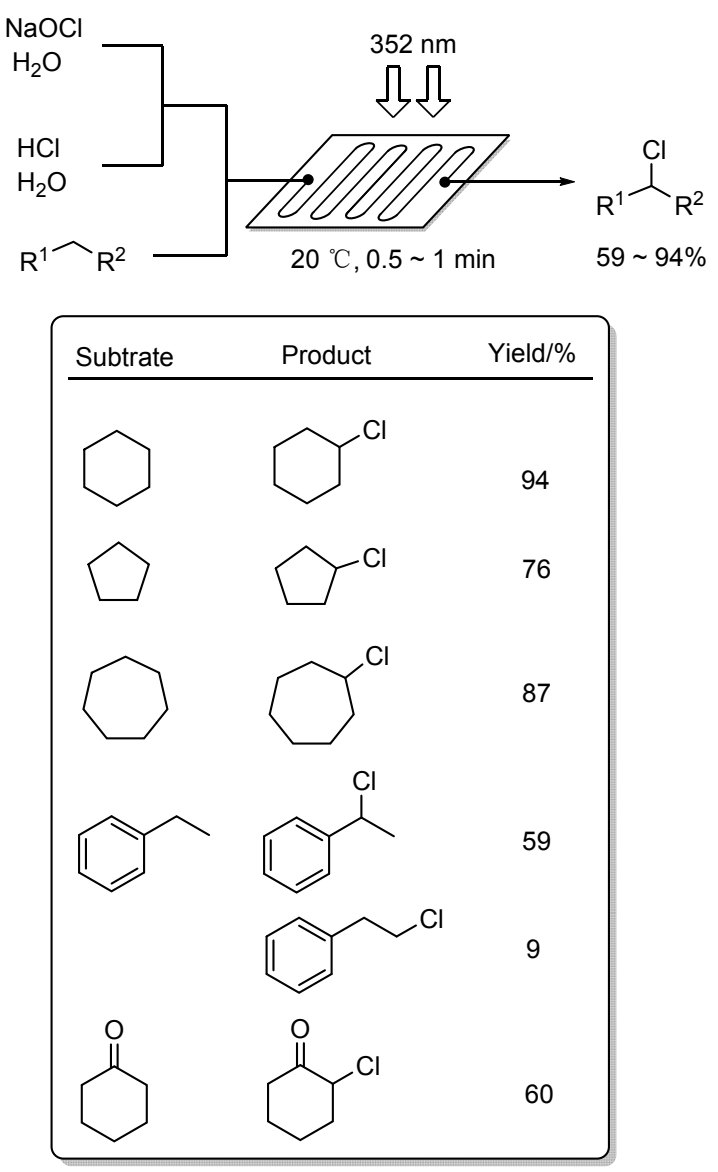

图式 9 利用原位生成的 $\mathrm{Cl}_{2}$ 对烃类进行光化学氯化

Scheme 9 Photochemical chlorination of hydrocarbons using in situ generated $\mathrm{Cl}_{2}$

Matsubara 等 ${ }^{[33]}$ 还报道了在光照条件下使用磺酰氯 $\left(\mathrm{SO}_{2} \mathrm{Cl}_{2}\right)$ 对环烷烃进行连续流自由基氯化反应(Scheme $10)$. 使用 $15 \mathrm{~W} 、 352 \mathrm{~nm}$ 的苂光灯辐射，对一系列环烷 烃在不同流速和停留时间下的反应进行了探索. 其中环 庚烷在流速为 $1 \mathrm{~mL} / \mathrm{h}$, 停留时间为 $57 \mathrm{~min}$ 的条件下收 率达到 $87 \%$. 反应过程中观察到产生的 $\mathrm{SO}_{2}$ 和 $\mathrm{HCl}$ 气体 
造成了塞流, 因此安装了背压调节器来抑制塞流. 实验 通过严格控制反应物物质的量比、光照强度和反应时间， 提高了反应的选择性, 单氯化产物的转化率大于多氯化 产物的转化率 ${ }^{[33]}$.

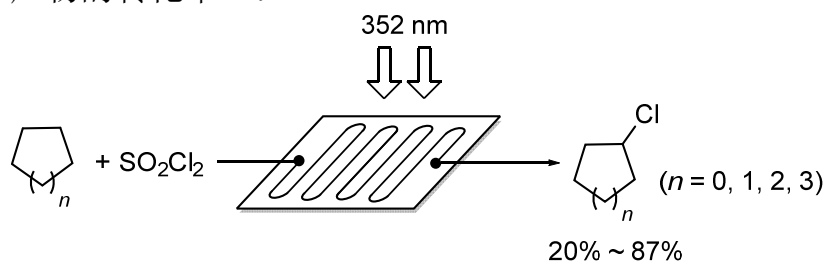

图式 10 硫酰氯微流氯化环烷烃

Scheme 10 Microflow chlorination of cycloalkanes with sulfuryl chloride

\section{3 流动化学与溴化反应}

溴化反应是有机合成中的一类重要的化学转化. 有 机溴化物的用途广泛, 可作为染料、阻然剂、药物活性 成分及聚合物的基本组成单位等. 将溴原子引入有机物 常用的试剂有 $\mathrm{Br}_{2} 、 \mathrm{HBr} 、 N$-溴代丁二酰亚胺(NBS)、溴 酸盐及次溴酸盐等 ${ }^{[34]}$. 使用 $\mathrm{Br}_{2}$ 对有机化合物进行溴化 主要是通过加成反应和取代反应实现. $\mathrm{Br}_{2}$ 是一种极具 腐蚀性和毒性的试剂, 易腐蚀设备和污染环境. 但是目 前 $\mathrm{Br}_{2}$ 由于其重要性依然在工业界被广泛应用, 开发其 安全利用的新技术十分重要.

Hessel 等 ${ }^{[35]}$ 于 2005 年报道了噻吩无溶剂连续溴化 反应(Scheme 11), 在室温下使用 $\mathrm{Br}_{2}$ 和芳香族化合物, 不使用任何催化剂. 该反应选择性很高, 相比传统釜式 反应，连续流反应得到的产物 2,5-二溴噻吩的收率明显 提高, 高达 $86 \%$. 此外, 反应时间也由大约 $2 \mathrm{~h}$ 缩短至 1 $\mathrm{s}$ 以内. 实验还比较了两种不同的微混合器, 内部混合 几何形状的微混合器实现了 $88 \%$ 的选择性, 而普通 $\mathrm{T}$ 型 混合器只有 72\%的选择性. 3-硝基甲苯的澳化和苯乙酮 的 $\alpha$-溴化也采用了类似方法得到了较高的产率 ${ }^{[36]}$. Fukuyama 等 ${ }^{[37]}$ 也报道了采用连续流反应技术进行烯烃的 溴化反应.

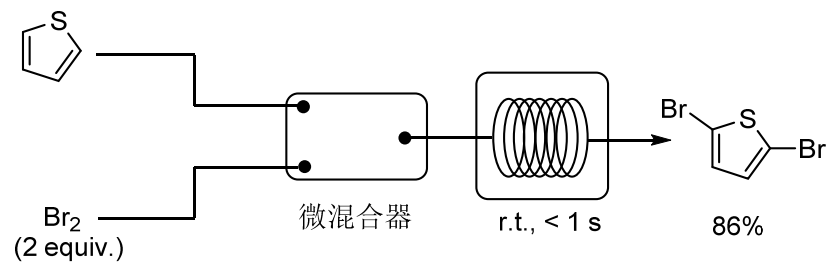

图式 11 噻吩的溴化反应

Scheme 11 Bromination reaction of thiophene

Stevens 等 ${ }^{[38]}$ 于 2014 年设计了一种连续流动反应系 统, 在相转移催化剂四丁基溴化铵(TBAB)的存在下, 将 $\mathrm{Br}_{2}$ 和 $\mathrm{KOH}$ 原位反应生成次溴酸钾来进行甲基砜或 甲磺酸盐的溴化(Scheme 12). 反应在较短的反应时间
内以 $99 \%$ 的产率生成了相应的三溴代化合物，在优化的 流动条件下, 产量可达 $53 \mathrm{~g} / \mathrm{d}$.

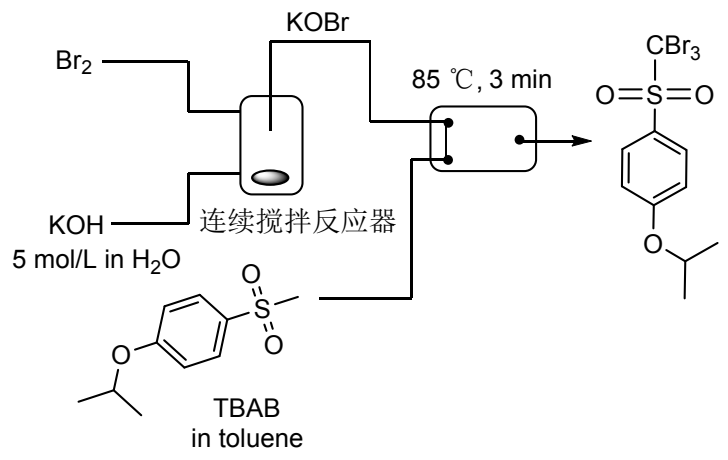

图式 12 原位生成 $\mathrm{KOBr}$ 溴化甲基砜和甲基磺酸盐 Scheme 12 In situ generation of $\mathrm{KOBr}$ for bromination of methylsulfones and methanesulfonates

$\mathrm{Ryu}$ 等 ${ }^{[39]}$ 于 2014 年报道了利用连续流光化学反应 对烷烃和烯烃进行溴化的工作(Scheme 13). 用 $15 \mathrm{~W}$, $352 \mathrm{~nm}$ 的荧光照射，反应抑制了二溴化物的生成，有效 地制备了高选择性的单溴化合物. 这种方法可应用于具 有光敏官能团的化合物 (如叠氮化合物) 的高效率溴 化 ${ }^{[39]}$.

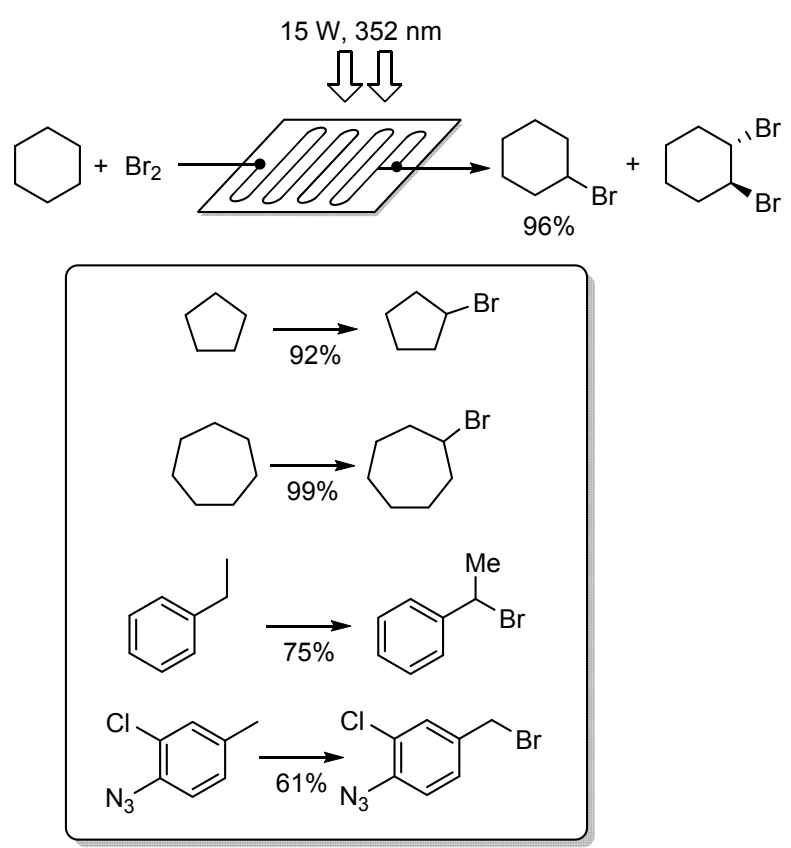

图式 13 烷烃和烯烃的溴化反应

Scheme 13 Bromination of alkanes and alkylarenes

Deng 等 ${ }^{[40]}$ 于 2016 年报道了芳基化合物的溴化反应 (Scheme 14). 实验将 1,2,4-三氟苯的 1,2-二氯乙烷(DCE) 溶液作为料液 1 通过玻璃注射器注入 $\mathrm{T}$ 型混合器, 将 $\mathrm{Br}_{2}$ 的 $\mathrm{DCE}$ 溶液作为料液 2 通过玻璃注射器注入，与铁 原位生成形成三溴化铁, 进入 $\mathrm{T}$ 型混合器, 混合后进入 
管式反应器，最后用 $\mathrm{Na}_{2} \mathrm{~S}_{2} \mathrm{O}_{3}$ 水溶液对反应混合物进行 淬灭. 该方法成功、稳定地合成了 2,4,5-三氟溴苯, 操作 简便，效率高，且反应只需几分钟，具有很好的工业化 前景 ${ }^{[40]}$.

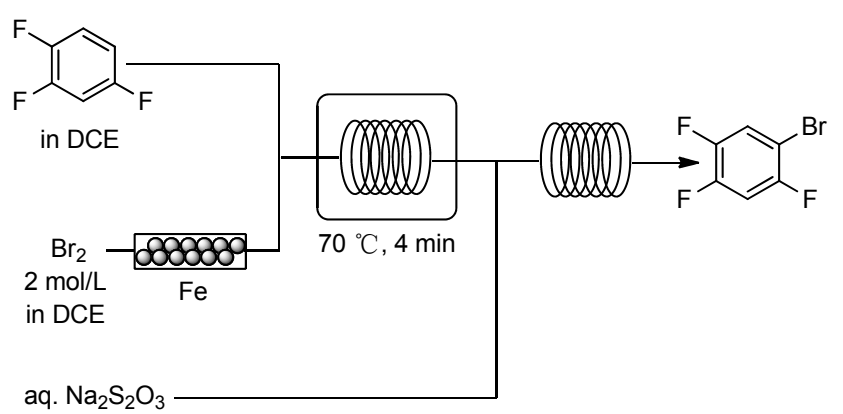

图式 14 1,2,4-三氟苯的溴化反应

Scheme 14 Bromination reaction of 1,2,4-trifluorobenzene

溴化氢亦常用于合成各种有机溴化物. 张跃等 ${ }^{[4]}$ 于 2013 年报道了微通道反应器中叔丁醇的溴化反应制 备溴代叔丁烷的工艺, 考察了原料物质的量配比、反应 温度、催化剂浓硫酸的用量及停留时间等单因素对反应 的影响. 通过实验结果得到了较佳的工艺条件: 反应温 度为 $50{ }^{\circ} \mathrm{C}$, 叔丁醇、氢溴酸和浓硫酸的物质的量比为 $1: 1.5: 1$, 停留时间 $60 \mathrm{~s}$, 叔丁醇的转化率为 $92 \%$, 溴 代叔丁烷的选择性为 $99 \%$, 而传统间歇摚拌反应器中叔 丁醇转化率为 $88 \%$, 溴代叔丁烷选择性为 $87 \%$. 相较于 传统工艺方法, 利用微通道技术降低了反应条件的要 求, 减少了废酸的产生, 大大提高了反应选择性和生产 效率.

NBS 常可代替 $\mathrm{Br}_{2}$ 对有机化合物进行溴化反应, 可 对烯烃、烯丙基类与苯甲基类化合物、羰基衍生物、芳 香类化合物进行溴化. NBS 较 $\mathrm{Br}_{2}$ 安全, 但进行溴化反 应时会产生等量的琥珀酰亚胺副产物, 甚至可能在连续 流动条件时导致堵塞. 为了避免这些问题. O'Brien 和 Cooper ${ }^{[22]}$ 于 2015 年利用在线液-液分离系统将澳化烯胺 酮时产生的副产物琥珀酰亚胺萃取到水流中, 无需进一 步纯化即可提供清洁产物. 实验将 $\mathrm{Na}_{2} \mathrm{~S}_{2} \mathrm{O}_{3}$ 和 $\mathrm{K}_{2} \mathrm{CO}_{3}$ 的 水溶液与反应混合物在二氯甲烷中混合. 过量的 NBS 被萃取到水相中淬灭, 琥珀酰亚胺也被萃取至水相中. 得到的有机相通过简单地蒸馏即可得到较高产率的溴 化物, 经过核磁共振氢谱检测未发现到琥珀酰亚胺的残 留(Scheme 15).

NBS 作为溴化剂的连续流技术也可用于光溴化反 应. Časar 等 ${ }^{[43]}$ 于 2013 年报道了瑞舒伐他汀前体化合物 5-甲基取代嘧啶的流动光化学溴化反应(Scheme 16). 传 统批量生产过程中反应物可以完全转化, 但是异构体和 副产物的生成无法避免且难于分离. 光澳化反应能更好

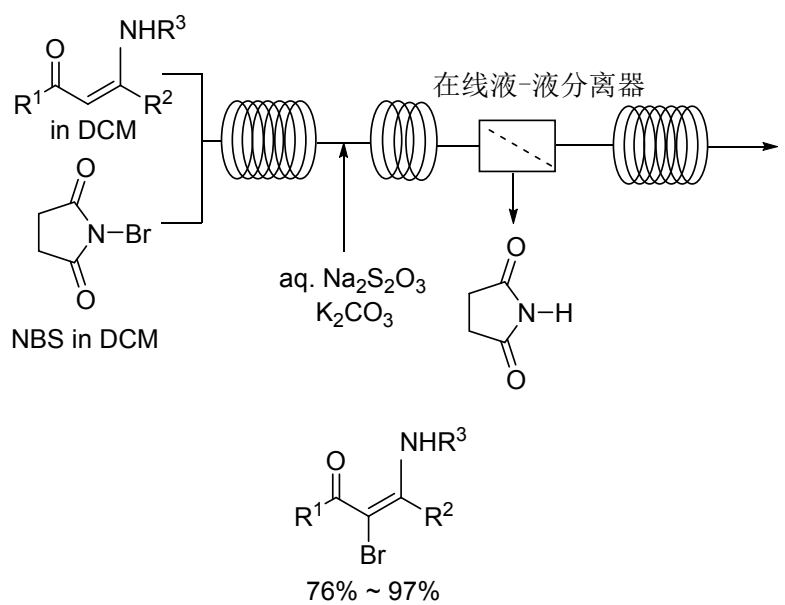

图式 15 NBS 溴化烯胺酮

Scheme 15 Bromination of enamine bromide using NBS

地控制溴化速率，抑制多溴化副产物地生成. 实验将氟 化乙烯丙烯共聚物(FEP)制成的毛细管缠绕在石英冷却 套上，用 $150 \mathrm{~W}$ 中压泵灯照射，反应停留时间为 $5 \mathrm{~min}$, 可获得 $58.3 \mathrm{mmol} / \mathrm{h}$ 的产物, 比传统批量生产的效率高 出近 4 倍. 5-芐基位置未发生溴化反应, 杂质总量较低.
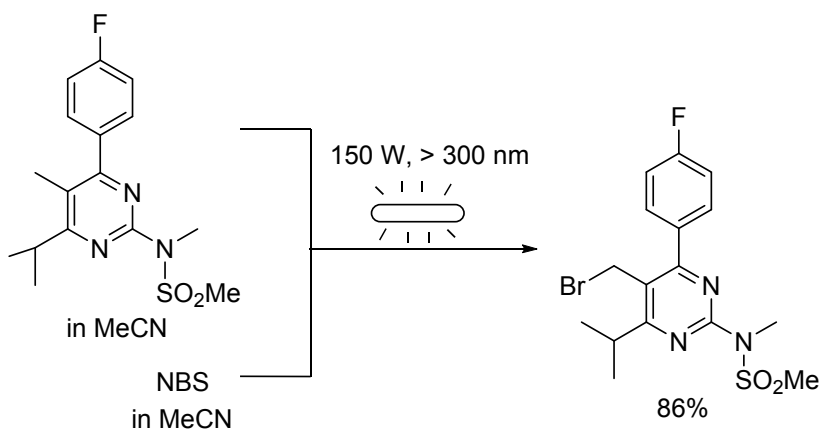

图式 16 5-甲基嘧啶的溴化反应

Scheme 16 Bromination of 5-methylpyrimidine

\section{4 流动化学与磑化反应}

有机碘化物作为合成原料或中间体广泛应用于多 类经典有机化学反应，包括亲核取代反应和 Suzuki 反 应、Stille 反应、Sonogashira 反应、Heck 反应等偶联反 应. 有机碘化物如甲状腺激素和三碘甲状腺素是人体必 须的化合物. 左旋甲状腺素是一种合成的甲状腺素，用 于治疗甲状腺功能衰退, 是全球最畅销的药物之一 ${ }^{[44]}$. 因此，有机化合物的碘化对于制备药物及其中间体具有 重要意义.

Yoshida 等 ${ }^{[45]}$ 于 2006 年首先报道了使用连续流微反 应对芳族化合物进行选择性单碘化(Scheme 17). 首先, $\mathrm{CH}_{3} \mathrm{CN}$ 中的 $\mathrm{I}_{2}$ 发生电解生成 $\mathrm{I}^{+}$, 得到的高活性的 $\left(\mathrm{CH}_{3} \mathrm{CN}\right)_{2} \mathrm{I}^{+}$对甲氧基苯进行亲电碘化. 将二甲氧基苯与 
电化学产生的 $\mathrm{I}^{+}$在单个微混合器中混合, 然后进一步进 入管式反应器反应. 微混合的速度极快, 对碘化的选择 性有利. 最终单碘化产物的收率提高至 $78 \%$ (传统釜式 反应单碘产物收率仅为 $45 \%$ ), 而二碘化物的收率则降 至 $4 \%$ (釜式为 $18 \%$ ).
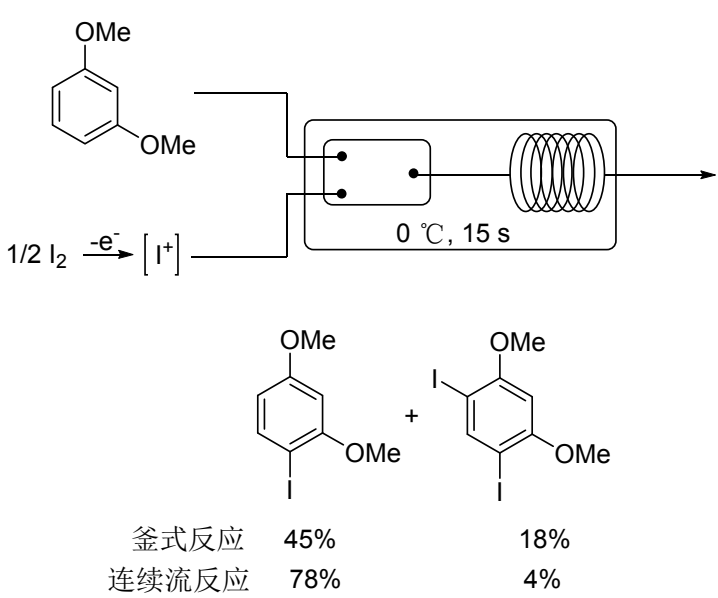

图式 17 连续流微反应器中 1,3-二甲基苯的选择性单碘化 Scheme 17 Selective monoiodination of 1,3-dimethoxybenene in continuous flow microreactor

Slocum 等 ${ }^{[46] 于 ~} 2011$ 年报道在连续流反应器中对芳 基胺进行的特异性碘化反应, 碘代产物经卤锂原位交换 后与二苯基甲酮反应生成三芳基甲醇产物, 总收率可以 达到 $63 \%$, 生产能力可以达到 $1.7 \mathrm{~kg} / \mathrm{h}$. 与传统釜式反 应相比具有更好的安全性、操作便捷性和可放大性. $D^{\prime}$ Attoma 等 $^{[47]}$ 于 2016 年也报道了使用 $I_{2}$ 直接碘化芳香 族化合物, 可用于制备 3-碘吲哚化合物(Scheme 18). 料 液 1 (含反应底物、 $\mathrm{Et}_{3} \mathrm{~N}$ 和 $\left.\mathrm{DMF}\right)$ 和料液 $2\left(\mathrm{I}_{2}\right.$ 的 $\mathrm{DMF}$ 溶 液, 1.1 equiv.)经 $\mathrm{T}$ 型混合器进入内径为 $1 \mathrm{~mm}$ 的管式反 应器, 在室温下仅停留 $1 \mathrm{~min}$, 最后用 $\mathrm{Na}_{2} \mathrm{~S}_{2} \mathrm{O}_{3}$ 及 $\mathrm{NH}_{4} \mathrm{OH}$ 水溶液对反应混合物进行淬灭. 反应得到的 3碘吲哚类化合物，收率高达 84\% 99\%.

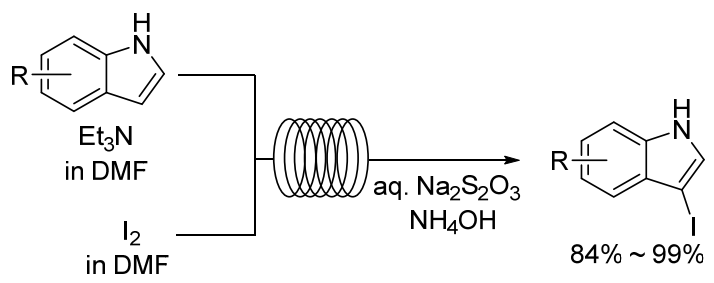

图式 18 吲哚的连续流碘代反应

Scheme 18 Continuous flow iodination of indoles

Ferreri 等 ${ }^{[48]}$ 于 2016 年改进先前开发的间歇碘化反 应, 将其在自主设计的多射流振荡盘(MJOD)流动反应 器上实现了百克级合成规模(Scheme 19). 该研究将反 应底物 5,5-二甲基海因和 $\mathrm{K}_{2} \mathrm{CO}_{3}$ 的水溶液以及 $\mathrm{EtOAc}$ 中的 $\mathrm{ICl}$ 组成的两相系统引入管式反应器, 反应温度为
$10{ }^{\circ} \mathrm{C}$, 停留时间为 $9 \mathrm{~min}$, 生产率为 $47 \mathrm{~g} / \mathrm{h}$. 将反应器 流出的产物过滤, 反应 $8 \mathrm{~h}$ 得到了 $375 \mathrm{~g}$ 纯产物 1,3-二 碘-5,5-二甲基海因(DIH), 收率约 90\%. DIH 可以用于芳 基化合物的碘化，是一种实用的碘化试剂.

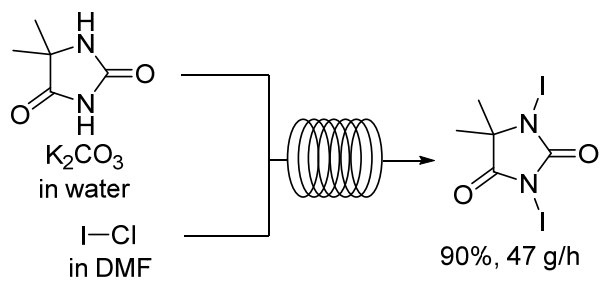

图式 19 用 ICl 对有机化合物进行碘化

Scheme 19 Iodination of organic compounds using ICl

\section{2 总结与展望}

随着绿色化学的发展，化学家们都在寻找更安全、 更高效且更环保的新反应技术，流动化学以其固有的优 势应运而生. 连续流动反应作为一种新兴反应技术，近 年来被广泛应用于医药、农药、化工产品及其中间体的 合成研究中, 并展现出巨大的应用潜力. 随着流动化学 理论和连续流反应技术的进步, 越来越多的连续流反应 设备被不断开发出来, 如微通道反应器、连续多级反应 器、旋转床反应器、连续流光化学反应器、连续流电化 学反应器及连续多级催化加氢反应器等. 如本文所述, 卤化反应明显是受益于流动化学发展的具有代表性的 例子. 高活性试剂 $\left(\mathrm{F}_{2} 、 \mathrm{Cl}_{2}\right.$ 等)可以安全、可控地参与合 成有机卤化物. 微反应器强化了传质, 试剂可被快速且 均匀地混合, 因此涉及使用 $\mathrm{F}_{2}$ 或 $\mathrm{Cl}_{2}$ 气体的气液反应提 高了反应效率和选择性. 在连续流反应中使用玻璃片或 透明管可以加速光化学反应, 例如用 $\mathrm{Cl}_{2}$ 进行的光化学 氯化反应以及 $\mathrm{Br}_{2}$ 和 $\mathrm{NBS}$ 参与的光化学溴化反应. 此外, 应用由耐腐蚀材料 $\mathrm{SiC}$ 或氟聚合物制作的微反应器，使 HF、 $F_{2}$ 这种极具腐蚀性的卤化试剂参与反应成为可能. 总的来说，将流动化学应用到卤化反应中，可以使危险 的卤化试剂以更可控的方式应用，可减少副产物的生 成，提高产率，降低能耗，缩短研发时间，提高原子经 济性，并强化生产过程. 对于多相卤代反应，可实现气一 液反应在流动中快速而充分的混合. 光化学卤化反应在 流动条件下可进行充分的辐照. 采用抗腐蚀材料制成的 微反应器允许使用具有极强腐蚀性的卤化试剂. 对于难 以储存、危害大或对空气、水敏感的卤化试剂还可以实 现原位生成和使用.

尽管连续流反应优势显著, 解决了传统釜式反应的 存在的一些问题. 但其进一步的应用和发展而言，仍面 临一些技术难题. 例如微反应器通道直径小, 流体流动 通常处于层流状态且表面张力作用显著, 微通道内气液 
传质的强化待进一步提升. 除了将微反应器通道设计成 特殊形状(弯折、碰撞等)来强化气液传质以外, 还可以 在尝试在微反应器中施加超声来强化传质. 处理和储存 大量具有毒性、腐蚀性的卤化试剂是卤化反应中重要的 安全问题, 开发更多可连续原位生成以供按需使用的卤 化试剂, 也是未来发展的方向之一. 此外, 虽然可通过 并联微反应器放大生产规模, 但相应的在线监测和控制 的难度都会随之增大, 同时所需的微反应器数量增多, 会提高合成工艺的成本. 在过去的几十年有机合成经历 了蓬勃发展, 今后如何利用微反应器的特点设计新型的 卤化反应是未来有机合成方法学的重要方向之一. 但毫 无疑问, 已崭露头角的流动化学工业应用成功范例正鼓 舞化学家和工程师们协同努力推进其更为广泛深入的 理论和应用研究.

\section{References}

[1] (a) Neumann, C. S.; Fujimori, D. G.; Walsh, C. T. Chem. Biol. 2008, $15,99$.

(b) Lin, R.; Amrute, A. P.; Perez-Ramirez, J. Chem. Rev. 2017, 117, 4182 .

[2] Veisi, H.; Ghorbani-Vaghei, R. Tetrahedron 2010, 66, 7445.

[3] Champagne, P. A.; Desroches, J.; Hamel, J. D.; Vandamme, M.; Paquin, J. F. Chem. Rev. 2015, 115, 9073.

[4] Wegner, J.; Ceylan, S.; Kirschning, A. Chem. Commun. 2011, 47, 4583.

[5] Plutschack, M. B.; Pieber, B.; Gilmore, K.; Seeberger, P. H. Chem. Rev. 2017, 117, 11796.

[6] (a) Suryawanshi, P. L.; Gumfekar, S. P.; Bhanvase, B. A.; Sonawane, S. H.; Pimplapure, M. S. Chem. Eng. Sci. 2018, 189, 431.

(b) Yao, X.; Zhang, Y.; Du, L.; Liu, J.; Yao, J. Renewable Sustainable Energy Rev. 2015, 47, 519.

(c) Pennemann, H.; Hessel, V.; Löwe, H. Chem. Eng. Sci. 2004, 59, 4789.

[7] (a) Cheng, D; Chen, F. Chem. Ind. Eng. Prog. 2019, 38, 556 (in Chinese).

(程荡，陈芬儿，化工进展, 2019, 38, 556.)

(b) Porta, R.; Benaglia, M.; Puglisi, A. Org. Process Res. Dev. 2016, 20, 2.

(c) Gutmann, B.; Cantillo, D.; Kappe, C. O. Angew. Chem., Int. Ed. Engl. 2015, 54, 6688.

[8] (a) Yu, T.; Ding, Z.; Nie, W.; Jiao, J.; Zhang, H.; Zhang, Q.; Xue, C.; Duan, X.; Yamada, Y. M. A.; Li, P. Chem.-Eur. J. 2020, 26, 5729 .

(b) Mak, X. Y.; Laurino, P.; Seeberger, P. H. Beilstein J. Org. Chem. 2009, 5, 19 .

[9] (a) Colella, M.; Carlucci, C.; Luisi, R. Top. Curr. Chem. 2018, 376. (b) Yu, T.; Jiao, J.; Song, P.; Nie, W.; Li, P. ChemSusChem 2020.

(c) Tanimu, A.; Jaenicke, S.; Alhooshani, K. Chem. Eng. J. 2017, $327,792$.

[10] Tucker, J. W.; Zhang, Y.; Jamison, T. F.; Stephenson, C. R. Angew. Chem., Int. Ed. 2012, 51, 4144.

[11] (a) Atobe, M.; Tateno, H.; Matsumura, Y. Chem. Rev. 2018, 118, 4541 .

(b) Tien, T. T.; Luu, T. L. Environ. Eng. Res. 2020, 25, 324.

[12] (a) Movsisyan, M.; Delbeke, E. I.; Berton, J. K.; Battilocchio, C.; Ley, S. V.; Stevens, C. V. Chem. Soc. Rev. 2016, 45, 4892. (b) Gao, Y..; Wang, J. Chin. J. Org. Chem. 2018, 38, 1275 (in Chinese). (郜云鹏; 王剑波，有机化学, 2018, 38, 1275.)

[13] Mason, B. P.; Price, K. E.; Steinbacher, J. L.; Bogdan, A. R.;
Mcquade, D. T. Chem. Rev. 2007, 107, 2300.

[14] (a) Illg, T.; Lob, P.; Hessel, V. Bioorg. Med. Chem. 2010, 18, 3707. (b) Bogdan, A. R.; Dombrowski, A. W. J. Med. Chem. 2019, 62, 6422 .

[15] (a) Tao, X.; Sheng, R.; Bao, K.; Wang, Y.; Jin, Y. Chin. J. Org. Chem. 2019, 39, 2726 (in Chinese).

(陶雪芬，盛荣，鲍勍，王玉新，金银秀，有机化学，2019，39， 2726.)

(b) Zhang, F.; Peng, X.; Ma, J. Chin. J. Org. Chem. 2019, 39, 109 (in Chinese).

(张发光, 彭星, 马军安, 有机化学, 2019, 39, 109.)

(c) Zhu, Y.-Y.; Wu, X.-D.; Abed, M.; Gu, S.-X.; Pu, L. Chem.-Eur. J. 2019, 25, 7866 .

[16] (a) Gillis, E. P.; Eastman, K. J.; Hill, M. D.; Donnelly, D. J.; Meanwell, N. A. J. Med. Chem. 2015, 58, 8315 .

(b) Wang. J.; Liu, H. Chin. J. Org. Chem. 2011, 31, 1785 (in Chinese). (王江, 柳红, 有机化学, 2011, 31, 1785.)

[17] (a) Campbell, M. G.; Ritter, T. Org. Process Res. Dev. 2014, 18, 474.

(b) Campbell, M. G.; Ritter, T. Chem. Rev. 2015, 115, 612.

(c) Lal, G. S.; Pez, G. P.; Syvret, R. G. Chem. Rev. 1996, 96, 1737.

[18] (a) Ma, J. A.; Cahard, D. Chem. Rev. 2004, 104, 6119.

(b) Shimizu, M.; Hiyama, T. Angew. Chem., Int. Ed. 2004, 44, 214.

(c) Richardson, P. Expert. Opin. Drug Discovery 2016, 11, 983.

(d) Zeng, L.; Mao, M.; Wang, L.; Zhang, X.; Ning, B. Fine Chem. 2019, 36, 549 (in Chinese).

(曾丽媛，毛明珍，王伦，张晓光，宁斌科，精细化工，2019，36, 549.)

[19] Chambers, R. D.; Spink, R. C. H. Chem. Commun 1999, 883.

[20] Chambers, R. D.; Hutchinson, J.; Sparrowhawka, M. E.; Sandforda, G.; Moilliet, J. S.; Thomson, J. J. Fluorine Chem. 2000, 102, 169.

[21] Chambers, R. D.; Sandford, G.; Trmcic, J.; Okazoe, T. Org. Process Res. Dev. 2008, 12, 339.

[22] Elgue, S.; Conte, A.; Gourdon, C.; Bastard, Y. Chim. Oggi 2012, 30, 18.

[23] Baumann, M.; Baxendale, I. R.; Martin, L. J.; Ley, S. V. Tetrahedron 2009, 65, 6611.

[24] Nagaki, A.; Uesugi, Y.; Kim, H.; Yoshida, J. Chem.-Asian J. 2013 , 8,705 .

[25] Naumann, K. Pest Manage. Sci. 2000, 56, 3.

[26] Schmittinger, P.; Florkiewicz, T.; Curlin, L. C.; Lüke, B.; Scannell, R.; Navin. T.; Zelfel, E.; Bartsch, R. Chlorine in Ullmann's Encyclopedia of Industrial Chemistry, Wiley-VCH Verlag, Weinheim, 2001.

[27] Strauss, F. J.; Cantillo, D.; Guerra, J.; Kappe, C. O. React. Chem. Eng. 2016, 1, 472 .

[28] Blacker, A. J.; Jolley, K. E. Beilstein J. Org. Chem. 2015, 11, 2408.

[29] Movsisyan, M.; Heugebaert, T. S. A.; Roman, B. I.; Dams, R.; Van Campenhout, R.; Conradi, M.; Stevens, C. V. Chem.-Eur. J. 2018, 24, 11779.

[30] Reichart, B.; Tekautz, G.; Kappe, C. O. Org. Process Res. Dev. 2012, 17, 152

[31] Borukhova, S.; Noël, T.; Hessel, V. Org. Process Res. Dev. 2016, 20,568

[32] Fukuyama, T.; Tokizane, M.; Matsui, A.; Ryu, I. React. Chem. Eng. 2016, 1,613 .

[33] Matsubara, H.; Hino, Y.; Tokizane, M.; Ryu, I. Chem. Eng. J. 2011, $167,567$.

[34] (a) Saikia, I.; Borah, A. J.; Phukan, P. Chem. Rev. 2016, 116, 6837.

(b) Groweiss, A. Org. Process Res. Dev. 2000, 4, 30.

(c) Steiner, A.; Williams, J. D.; de Frutos, O.; Rincón, J. A.; Mateos, C.; Kappe, C. O. Green Chem. 2020, 22, 448.

[35] Löb, P.; Hessel, V.; Klefenz, H. Lett. Org. Chem. 2005, 2, 767.

[36] (a) Löb, P.; Löwe, H.; Hessel, V. J. Fluorine Chem. 2004, 125, 1677.

(b) Becker, R.; van den Broek, S. A. M. W.; Nieuwland, P. J.; Koch, K.; Rutjes, F. P. J. T. J. Flow Chem. 2012, 2, 87. 
[37] Fukuyama, T.; Rahman, M. T.; Kamata, N.; Tokizane, M.; Fukuda, Y.; Ryu, I. J. Flow Chem. 2013, 3, 4.

[38] Van Waes, F. E. A.; Seghers, S.; Dermaut, W.; Cappuyns, B.; Stevens, C. V. J. Flow Chem. 2014, 4, 118.

[39] Manabe, Y.; Kitawaki, Y.; Nagasaki, M.; Fukase, K.; Matsubara, H.; Hino, Y.; Fukuyama, T.; Ryu, I. Chem.-Eur. J. 2014, 20, 12750.

[40] Deng, Q.; Shen, R.; Ding, R.; Zhang, L. Chem. Eng. Technol. 2016, $39,1445$.

[41] Zhang, Y.; Guo, X.; Yan, S.; Liu, J.; Shen, J. Spec. Petrochem. 2013, 30, 58 (in Chinese).

(张跃, 郭欣桐, 严生虎, 刘建武, 沈介发, 精细石油化工, 2013, 30, 58.)
[42] O’Brien, M.; Cooper, D. Synlett 2015, 27, 164.

[43] Šterk, D.; Jukič, M.; Časar, Z. Org. Process Res. Dev. 2013, 17, 145.

[44] Cantillo, D.; Kappe, C. O. React. Chem. Eng. 2017, 2 , 7.

[45] Midorikawa, K.; Suga, S.; Yoshida, J. Chem. Commun. 2006, 3794.

[46] Slocum, D. W.; Tekin, K. C.; Nguyen, Q.; Whitley, P. E.; Reinscheld, T. K.; Fouzia, B. Tetrahedron Lett. 2011, 52, 7141.

[47] D'Attoma, J.; Cozien, G.; Brun, P. L.; Robin, Y.; Bostyn, S.; Buron, F.; Routier, S. ChemistrySelect 2016, 1, 338.

[48] Ferreri, M.; Drageset, A.; Gambarotti, C.; Bjørsvik, H.-R. React. Chem. Eng. 2016, 1, 379.

(Cheng, F.) 